\title{
GENERALIZED RANDOM PROCESSES AND CAUCHY'S PROBLEM FOR SOME PARTIAL DIFFERENTIAL SYSTEMS
}

\section{MAHMOUD M. EL-BORAI}

Faculty of Science King Abdulaziz University

P.O. Box 1540 Jeddah

SAUDI ARABIA

(Received February 1, 1979 and in revised form October 22, 1979)

ABSTRACT. In this paper we consider a parabolic partial differential system of the form $D_{t} H_{t}=L(t, x, D) H_{t}$. The generalized stochastic solutions $H_{t}$, corresponding to the generalized stochastic initial conditions $\mathrm{H}_{0}$, are given. Some properties concerning these generalized stochastic solutions are also obtained.

KEY WORDS AND PHRASES. Generalized Stochastic Solutions, Strongly Parabolic Systems.

1980 MATHEMATICS SUBJECT CLASSIFICATION CODES: $60 H 15,35 R 60$.

1. INTRODUCTION.

Consider the system

$$
D_{t} u=L u
$$


where

$$
\begin{gathered}
D_{t}=\frac{\partial}{\partial t}, L=\sum_{|k| \leq 2 b} L_{k}(t, x) D^{k}, \\
D^{k}=(-i)^{k} D_{1}^{k} \ldots D_{n}^{k}, D_{r}=\frac{\partial}{\partial x_{r}}, r=1, \ldots n,
\end{gathered}
$$

$|k|=k_{1}+\ldots+k_{n}, t \in(0, T), T>0, x$ is an element of the $n$-dimensional

Euclidean space $E_{n}$, and $\left(L_{k}(t, x),|k| \leq 2 b\right)$ is a family of square matrices of order N.

We assume that $(1.1)$ is a strongly parabolic system on $G_{n+1}=\{(t, x)$ : $\left.t \in[0, T], x \in E_{n}\right\}$ in the sense that for every complex vector $a=\left(a_{1}, \ldots, a_{N}\right)$, every $\phi \in E_{n}$, and every $(t, x) \in G_{n+1}$;

$$
\operatorname{Re}\left[\sum_{|k|=2 b} L_{k}(t, x) \sigma^{k} a, \bar{a}\right] \leq-\delta|\sigma|^{2 b}|\alpha|^{2}
$$

where

$$
\sigma^{k}=\sigma_{1}^{k_{1}} \ldots \sigma_{n}^{k_{n}},|\sigma|^{2 b}=\left(\sigma_{1}^{2}+\ldots+\sigma_{n}^{2}\right)^{b},
$$

$|a|^{2}=a_{1}^{2}+\ldots+a_{N}^{2}$, and $\delta$ is a positive constant (see [1]). In the above inequality and in the following, we denote the scalar product of two $\mathrm{N}$-vector functions $\mathrm{u}$ and $\mathrm{v}$ by the bracket notation $(\mathrm{u}, \mathrm{v})$.

As usual, we denote by $c^{m}\left(E_{n}\right), 0 \leq m \leq \infty$, the set of all real-valued functions defined on $E_{n}$, which have continuous partial derivatives of order up to and including $m$ (of order $<\infty$ if $m=\infty$ ). By $C_{0}^{m}\left(E_{n}, N\right)$ we denote the set of all vector functions $h=\left(h_{1}, \ldots, h_{N}\right)$ such that every $h_{r}$ is in $c^{m}\left(E_{n}\right)$, with compact support, $r=1, \ldots, N$. We assume that the elements of the matrices $L_{k}(t, x),|k| \leq 2 b$, satisfy the following conditions:

(a) They are bounded on $G_{n+1}$ and satisfy a Holder condition of order $\alpha$ with respect to $\mathrm{x},(0<\alpha \leq 1)$.

(b) For every $x \in E_{n}$, they are continuous functions in $t \in[0, T]$. 
(c) For every $t$ in $[0, T]$, they are $C^{\infty}\left(E_{n}\right)$ functions. Let $u=$ $\left(u_{1}, \ldots, u_{N}\right)$ satisfy the initial condition

$$
u(x, 0)=u_{0}(x) \text {, }
$$

where $u_{0}=\left(u_{o 1}, \ldots, u_{o N}\right),\left[u_{o r} \in C\left(E_{n}\right)\right.$ are bounded on $\left.E_{n}, r=1, \ldots, N\right]$. We say that $u$ is of the class $S\left(E_{n}\right)$ if for each $t \in(0, T), D_{t} u_{r} \in C\left(E_{n}\right)$ and $u_{r} \in C^{2 b}\left(E_{n}\right), r=1, \ldots, N$.

It has been proved [2] that, under conditions (a) and (b), there exists a fundamental matrix $\mathrm{Z}(\mathrm{t}, 0, \mathrm{x}, \mathrm{y})$ of the system (1.1) such that

$$
u(t, x)=\int_{E_{n}} z(t, 0, x, y) u_{o}(y) d y, d y=d y_{1} \ldots d y_{r}
$$

represents the unique solution of the Cauchy problem (1.1), (1.2) in the class $S\left(E_{n}\right)$

Let $\left(V_{r}: r=1, \ldots, N\right)$ be a family of Gaussian random measures in the sense of Gelfand and Vilenkin [2]. Let $g_{r}$ be a complex-valued function defined on $E_{1}$. We say that $g_{r}$ is of the class $K_{r}$ if the integral

$$
\begin{aligned}
& \left.\int_{\mathrm{E}_{1}} \lg _{\mathrm{r}}(\mathrm{s})\right|^{2} \mathrm{dF} \mathrm{r}_{\mathrm{r}}(\mathrm{s}) \text { exists, where } \mathrm{F}_{\mathrm{r}} \text { is a positive measure such that } \\
& E\left[v_{r}\left(B_{1}\right) \overline{v_{r}\left(B_{2}\right)}\right]=F_{r}\left(B_{1} \cap B_{2}\right)
\end{aligned}
$$

for any two Borel sets $B_{1}$ and $B_{2}$ on the real line $[r=1, \ldots, N$ and $E()$. denotes the expectation of $()$.$] .$

Let $\mathrm{H}$ be an $\mathrm{N}$-vector of generalized stochastic processes, which associates with every $h$ in $C_{0}^{\infty}\left(E_{n}, N\right)$ an $N$-vector of random variables defined by

$$
\begin{aligned}
& H(h)=\left(H_{1}(h), \ldots, H_{N}(h)\right), \\
& H_{r}(h)=\int_{E_{1}} g_{r o}(s) d V_{r}(s),
\end{aligned}
$$




$$
g_{r o}(s)=\int_{E_{n}}\left(I_{r}(x, s), h(x)\right) d x,
$$

where $\left(I_{r} ; r=1, \ldots, N\right)$ is a family of $N$-vectors of continuous functions on $\mathrm{E}_{\mathrm{n}+1}$.

It is assumed also that all the components of $I_{r}$ are bounded on $E_{n}$, independently of s. Clearly, $g_{\text {ro }}$ is of the class $\mathrm{K}_{\mathrm{r}}$.

The theoretical development in section 2 exhibits the use of formula (1.3) in order to integrate (1.1) when the initial condition is an N-vector of generalized stochastic processes, which is defined by (1.4). Also, some essential properties are derived in section 3.

\section{GENERALIZED STOCHASTIC SOLUTIONS.}

An $N$-vector $w(t, x, s)$ of functions is said to be of the class $C\left(E_{n+1}, N\right)$ if, for each $t$ in $(0, T)$, the components of $w(t, x, s)$ represent continuous functions of $(x, s)$ on $E_{n+1}$ and they are bounded on $E_{n}$, independently of $s$. We say that the generalized stochastic vector $\mathrm{H}_{t}$ is of the class $\mathrm{V}$ if there exists a family $\left[s_{r}(t, x, s): S_{r} \in C\left(E_{n+1}, N\right), r=1, \ldots, N\right]$ such that, for each $h$ in $C_{0}^{\infty}\left(E_{n}, N\right), H_{t}(h)$ can be represented in the form

$$
\begin{aligned}
& H_{t}(h)=\int_{E_{1}} g(t, s) d V(s), \\
& g=\left(g_{1}, \ldots, g_{N}\right), g_{r}(t, s)=\int_{E_{n}}\left(S_{r}(t, x, s), h(x)\right) d x, \\
& H_{t}(h)=\left(H_{1 t}(h), \ldots, H_{N t}(h)\right), H_{r t}(h)=\int_{E_{1}} g_{r}(t, s) d V_{r}(s) .
\end{aligned}
$$

It is clear that, for each $t$ in $(0, T), g_{r} \mid \in K_{r}$. The expectation of $\left|H_{r t}\right|^{2}$ is given by 


$$
E\left|H_{r t}\right|^{2}=\int_{E_{1}}\left|g_{r}(t, s)\right|^{2} d F_{r}(s) .
$$

If $D_{t} g_{r}(t, s)$ exists and belongs to $K_{r}$ for each $t$ in $(0, T)$, then we define $\frac{d}{d t} H_{r t}(h)$ by

$$
\frac{d}{d t} H_{r t}(h)=\underset{\substack{1 . i . m \\ t \rightarrow 0}}{ } \int_{E_{1}} \frac{\Delta g_{r}(t, s)}{\Delta t} d v_{r}(s)=\int_{E_{1}} D_{t} g_{r}(t, s) d v_{r}(s),
$$

where $\Delta g_{r}(t, s)=g_{r}(t+\Delta t, s)-g_{r}(t, s)$ and 1.i.m. denotes limit in the mean, 1.e.

$$
\lim _{t \rightarrow 0} \int_{E_{1}}\left|\frac{\Delta g_{r}(t, s)}{\Delta t}-D_{t} g_{r}(t, s)\right|^{2} d_{r}(s)=0
$$

Let $L^{*}=\sum_{|k| \leq 2 b}(-1)|k| D^{k} L_{k}^{*}$, where $\left(L_{k}^{*},|k| \leq 2 b\right)$ is the family of adjoint matrices to $\left(L_{k},|k| \leq 2 b\right)$. Since the coefficients of the operator $L$ are $C^{\infty}\left(E_{n}\right)$ functions, it follows that, for every $h$ in $C_{0}^{\infty}\left(E_{n}, N\right), L^{*} h=h_{t}$ 1s also in $C_{0}^{\infty}\left(E_{n}, N\right)$. We call $H_{t}$ a generalized stochastic solution of the system (1.1) if $H_{t}$ and $\frac{\mathrm{dH}_{t}}{\mathrm{dt}}$ are of the class $\mathrm{V}$ and

$$
{\frac{d H_{t}}{d t}}^{(h)}=H_{t}\left(h_{t}^{*}\right)
$$

for every $h$ in $C_{0}^{\infty}\left(E_{n}, N\right)$ and $t$ in $(0, T)$. We assume that

$$
H_{0}(h)=H(h)
$$

where $\mathrm{H}$ is defined by (1.4).

THEOREM 1: The Cauchy problem $(2.1),(2.2)$ has a unique generalized stochastic solution $H_{t}$ in the class $V$.

PROOF: Let $\left(S_{r}(t, x, s): r=1, \ldots, N\right)$ be a family of solutions of the system (1.1) with the initial conditions: 


$$
S_{r}(0, x, s)=I_{r}(x, s), r=1, \ldots, N
$$

Using formula (1.3), one gets

$$
S_{r}(t, x, s)=\int_{E_{n}} Z(t, 0, x, y) I_{r}(y, s) d y .
$$

According to the properties of the fundamental matrix $z$, we find $s_{r^{\prime}} \in C\left(E_{n+1}, N\right)$, $\mathrm{r}=1, \ldots, \mathrm{N}$. Set,

$$
H_{t}(h)=\int_{E_{1}} g(t, s) d V(s)
$$

and

$$
g_{r}(t, s)=\int_{E_{n}}\left(s_{r}(t, x, s), h(x)\right) d x \text { with } h \in C_{0}^{\infty}\left(E_{n}, N\right),
$$

where $s_{1}(t, x, s), \ldots, s_{N}(t, x, s)$ are defined by $(2.3)$. Since $s_{r} \in C\left(E_{n+1}, N\right)$, it follows that $H_{t}$ is of the class $V$. Using again the properties of $Z$, we get

$$
\begin{aligned}
D_{t} \int_{E_{n}}\left(S_{r}(t, x, s), h(x)\right) d x & =\int_{E_{n}}\left(D_{t} S_{r}(t, x, s), h(x)\right) d x \\
& =\int_{E_{n}}\left(S_{r}(t, x, s), h_{t}^{*}(x)\right) d x .
\end{aligned}
$$

The last formula proves that $D_{t} g_{r^{\prime}} \in K_{r}$.

Now we already have

$$
\frac{d}{d t} H_{t}(h)=\int_{E_{1}} \int_{E_{n}}\left(S_{r}(t, x, s), h_{t}^{*}(x)\right) d x d V(s)=H_{t}\left(h_{t}^{*}\right),
$$

where $\frac{d}{d t} H_{t}$ is of the class $v$.

We also have

$$
H_{0}(h)=\int_{E_{1}} g(0, s) d V(s) \text {, }
$$


where

$$
g_{r}(0, s)=\int_{E_{n}}\left(I_{r}(x, s), h(x)\right) d x
$$

Thus the existence of the generalized stochastic solution $H_{t}$ with the initial condition $\mathrm{H}_{\mathrm{O}}=\mathrm{H}$ is proved. To prove the uniqueness of $\mathrm{H}_{t}$, it is sufficient to show that the only solution of $(2.1)$ with the initial condition $H_{0}(h)=$ $H(h)=0$ is $H_{t}(h)=0$ for every $h$ in $C_{0}^{\infty}\left(E_{n}, N\right)$ and $t$ in $(0, T)$. If $H_{0}=0$, then $E\left|H_{\text {ro }}\right|^{2}=\int_{E_{1}}\left|g_{\text {ro }}(s)\right|^{2} \mathrm{dF}(s)=0$, and hence $g_{\text {ro }}(s)=0$ on $E_{1}$. Therefore,

$$
g_{r o}(s)=\int_{E_{n}}\left(I_{r}(x, s), h(x)\right) d x=0,
$$

which is true for any arbitrary $h$ in $C_{0}^{\infty}\left(E_{n}, N\right)$, and hence $I_{r}(x, s)=0$ on $E_{n+1}$. Since $\frac{d}{d t} H_{t}(h)=H_{t}\left(h_{t}^{*}\right)$, it follows that

$$
E\left|\frac{d}{d t} H_{r t}(h)-H_{r t}\left(h_{t}^{*}\right)\right|^{2}=0 \text {; }
$$

therefore,

$$
\int_{E_{n}}\left(D_{t} S_{r}(t, x, s)-L S_{r}(t, x, s), h(x)\right) d x=0,
$$

which implies

$$
D_{t} S_{r}(t, x, s)=L S_{r}(t, x, s) .
$$

We also have

$$
s_{r}(0, x, s)=0 .
$$

The uniqueness of the problem (2.4), (2.5) gives

$$
S_{r}(t, x, s)=0,
$$




$$
t_{1} \in(0, T),(x, s) \in E_{n+1},(r=1, \ldots, N)
$$

Using (2.6), one gets $H_{t}(h)=0$, for every $h$ in $C_{0}^{\infty}\left(E_{n}, N\right)$ and $t$ in $(0, T)$. This completes the proof.

\section{A CONVERGENCE THEOREM.}

Let $h_{m}=\left(h_{m_{1}}, \ldots, h_{m_{N}}\right), m=1,2, \ldots$ be a sequence in $c_{0}^{\infty}(G, N)$, where $G$ is a bounded open domain of $E_{n}$. Suppose that

$$
\lim _{m \rightarrow \infty} \int\left(h_{m_{r}}(x)-w_{r}(x)\right)^{2} d x=0
$$

where ${ }_{r} \in_{L_{2}}(G), r=1, \ldots, N$ and $L_{2}(G)$ denotes the set of all Lebesgue measurable square integrable functions on $G$. It is assumed that $w_{r}(x)=0$ for $x \in G$ where $r=1, \ldots N$.

THEOREM 2: If $\mathrm{H}_{\mathrm{t}}\left(\mathrm{h}_{\mathrm{m}}\right)=\int \mathrm{g}_{\mathrm{m}}(\mathrm{t}, \mathrm{s}) \mathrm{dV}(\mathrm{s})$,

then

$$
\underset{\mathrm{m} \rightarrow \infty}{\lim .} \mathrm{H}_{t}\left(\mathrm{~h}_{\mathrm{m}}\right)=\int \eta(t, s) \mathrm{dV}(\mathrm{s})
$$

where $g_{m}(t, s)=\left(g_{m_{1}}(t, s), \ldots, g_{m_{N}}(t, s)\right)$,

$$
\begin{aligned}
& g_{m_{r}}(t, s)=\int\left(s_{r}(t, x, s), h_{m}(x)\right) d x, \eta=\left(\eta_{1}, \ldots, \eta_{N}\right), \\
& n_{r}(t, s)=\int\left(s_{r}(t, x, s), w(x)\right) d x, \text { and the family }\left(s_{r}, r=1, \ldots, N\right)
\end{aligned}
$$

is defined by (2.3).

PROOF: A straight forward application of the Cauchy - Schwarz inequality establishes that

$$
\lim _{m \rightarrow \infty} \int_{G}\left(S_{r}(t, x, s), h_{m}(x)\right) d x=\int_{G}\left(S_{r}(t, x, s), w(x)\right) d x
$$


According to the conditions imposed on the family $\left(I_{r}(x, s), r=1, \ldots, N\right)$ and according to the properties of the fundamental matrix $\mathrm{Z}$, we can find a constant A such that

$$
\left|g_{m_{r}}(t, s)\right| \leq A
$$

for all $\mathrm{m}, \mathrm{s}, \mathrm{t}, \in(0, \mathrm{~T})$ and $\mathrm{r}=1, \ldots, \mathrm{N}$. For any positive integers $\ell$ and $\mathrm{m}$, we have

$$
E\left|H_{r t}\left(h_{m}\right)-H_{r t}(h)\right|^{2}=\int\left|g_{m_{r}}(t, s)-g_{\ell_{r}}(t, s)\right|^{2} d F_{r}(s) \text {. }
$$

By a standard argument based on (3.2) and (3.3), the righthand side of (3.4) can be shown to go to zero. Thus, $\mathrm{H}_{t}\left(\mathrm{~h}_{\mathrm{m}}\right)$ is a Cauchy sequence. We deduce also that

$$
\lim _{m \rightarrow \infty} \int\left|g_{m_{r}}(t, s)-n_{r}(t, s)\right|^{2} d F_{r}(s)=0
$$

The last argument leads to the fact that there exists a stochastic process $R_{r}(t)$ such that $E|R(t)|^{2}<\infty$ and that

$$
\lim _{m \rightarrow \infty} E\left|H_{r t}\left(h_{m}\right)-R_{r}(t)\right|^{2}=0
$$

Following Doob [3], we find

$$
\begin{aligned}
& R_{r}(t)=\int \eta_{r}(t, s) d v_{r}(s), \\
& \eta_{r}(t, s)=\int\left(S_{r}(t, x, s), w(x)\right) d x .
\end{aligned}
$$

This completes the proof.

COROLLARY: For vector functions $\left(\mathrm{w}=\mathrm{w}_{1}, \ldots, \mathrm{w}_{\mathrm{N}}\right)$ where $\mathrm{w}_{\mathbf{r}} \epsilon_{1} \mathrm{~L}_{2}(\mathrm{Q})$ and $w_{r}(x)=0$ for $\left.x \notin G\right)$, there exists a sequence $\left(h_{m}\right)$ in $C_{0}^{\infty}\left(E_{n}\right.$, $\left.N\right)$ such that

$$
\underset{m \rightarrow \infty}{1 . i . m .} H_{0}\left(h_{m}\right)=H_{o}(w),
$$




$$
\underset{m \rightarrow \infty}{\text { 1.i.m. }} \underset{t}{H}\left(h_{m}\right)=H_{t}(w) \text {. }
$$

The proof can be deduced direct1y by using theorem 2. (Compare [4]).

\section{REFERENCES}

[1] C.D. Idelman, Parabolic Systems. P. 87, Moscow 1964, (Mir Pub1ishers).

[2] N.Ya. Vilenkin, I.M. Gelfand, Generalized Functions, Vo1. 4, 1964 (Translated).

[3] J.L. Doob, Stochastic Processes. New York 1953.

[4] M.M. E1-Borai, On Stochastic Differential Equation in a Hilbert Space, Egyptian Statistical Journal Vo1. 17, No. 2, 1973. 


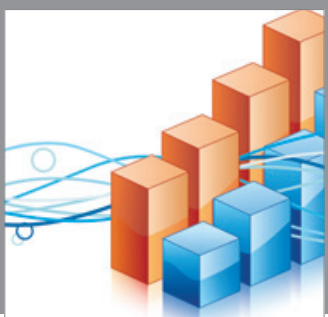

Advances in

Operations Research

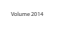

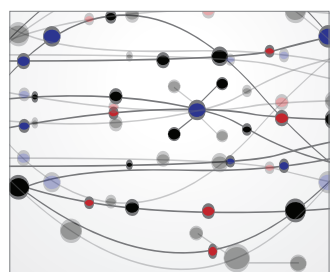

\section{The Scientific} World Journal
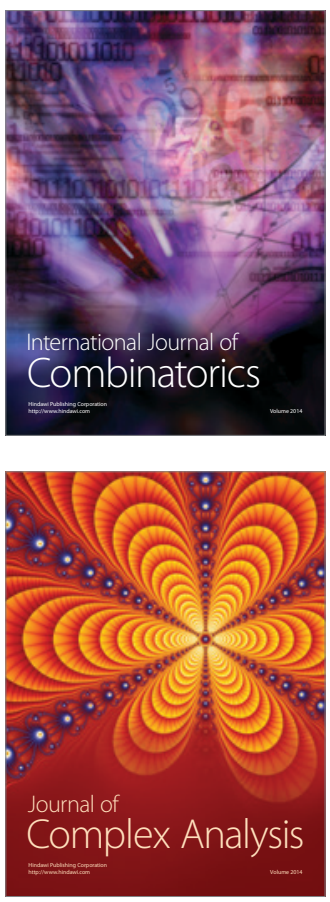

International Journal of

Mathematics and

Mathematical

Sciences
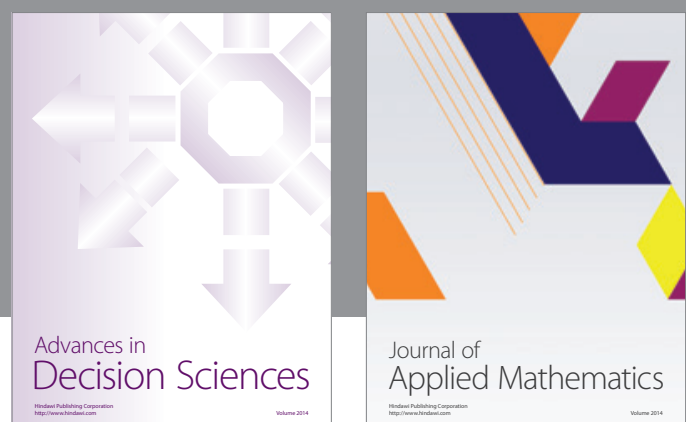

Journal of

Applied Mathematics
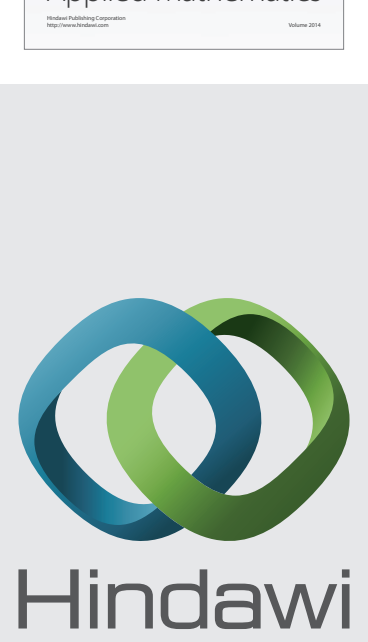

Submit your manuscripts at http://www.hindawi.com
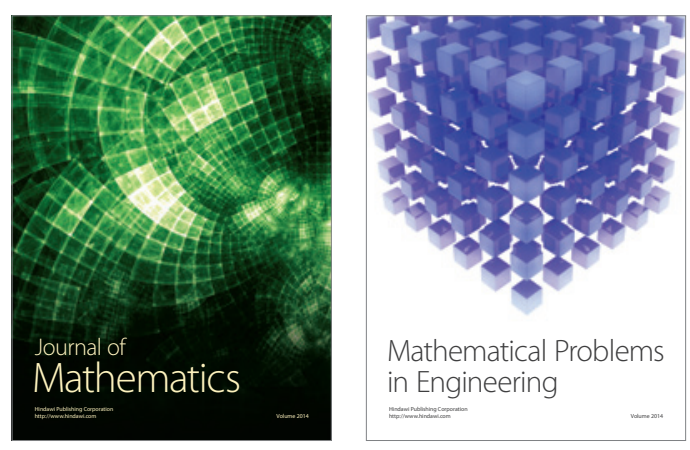

Mathematical Problems in Engineering
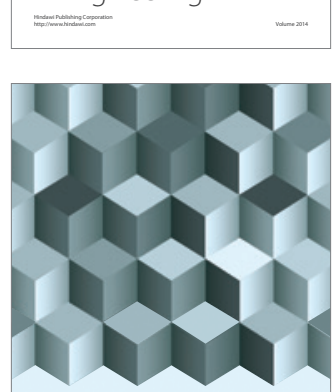

Journal of

Function Spaces
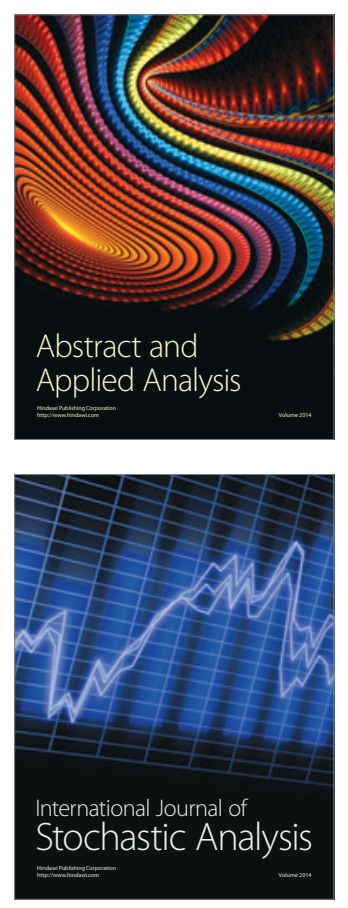

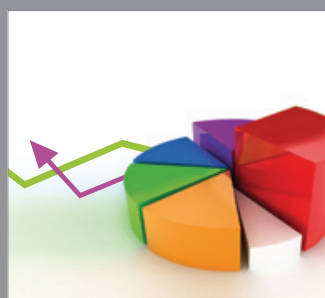

ournal of

Probability and Statistics

Promensencen
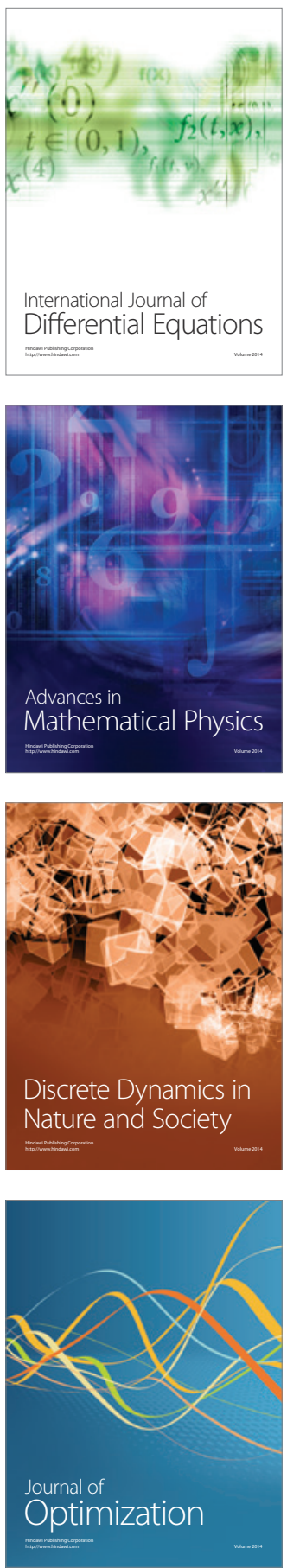